\title{
Supervisory Management and Lightpath Restoration for Wavelength Routing Networks
}

\author{
Frank Tong, Senior Member, IEEE, Tak-Shing Yum, Senior Member, IEEE, and Chi-Chun Hui
}

\begin{abstract}
We present here a supervisory management scheme and two complete lightpath restoration algorithms based on integer linear programming. The supervisory management uses a hierarchical approach with a network manager supervising all node managers, each of which monitors the health status of all network components and subsystems on that link. Extension to the existing CCS7 protocol is proposed to include the surveillance information in the signaling network. For the restoration algorithms, their performances are compared in three networks commonly cited for testing purposes. In general, the source-based restoration algorithm performs better than that based on the link-based restoration algorithm, but requires much longer computation time. Also, the former restoration algorithm ensures the fairness in satisfying lightpath demands within the network.
\end{abstract}

Index Terms-Lightpath restoration, supervisory management, wavelength routing networks.

\section{INTRODUCTION}

$\mathbf{T}$ TRANSPORT network based on all-optical wavelength-division multiple-access (WDMA) wavelength routing network [1] is probably by far the most viable architecture that can meet both bandwidth and node-scalability demands required in the future. At present, wavelength-division multiplexing (WDM) networking products are commercially available, and researchers worldwide are racing toward the realization of a practical, scalable, all-optical multi-access network. An area of particular interest lies in supervisory management and network restoration, where the surveillance information can be managed and processed, and any downtime arising from component and system failures can be minimized through traffic re-routing by assigning a backup lightpath between the source node and the destination node.

Although reports on supervisory management and restoration schemes for WDM transport networks are relatively few, many adaptations can be obtained from schemes previously investigated for electronic based networks. For example, a pool of backup paths [2], [3] is proposed to set aside for asynchronous transfer mode (ATM) networks. Another scheme [4] makes use of efficient signaling mechanism and fast switching. Several algorithms [5]-[7] based on integer linear programming were previously designed for traffic

Manuscript received June 15, 1999; revised May 2, 2000. The work was supported by RGC-CUHK 4157/98E from the Hong Kong SAR Government.

The authors are with the Department of Information Engineering, The Chinese University of Hong Kong, Shatin, N.T., Hong Kong (e-mail: fktong@ie.cuhk.edu.hk; tsyum@ie.cuhk.edu.hk).

Publisher Item Identifier S 0733-8724(00)08067-1. routing and network planning. For all-optical networks, lightpath routing algorithms were developed [8], [9], and related work on spare capacity planning is also reported in [10]. Here, we will focus on lightpath restoration algorithm designed for a single link failure. Also, we will propose a supervisory management scheme and a signaling protocol based on modifications of Common Channel Signaling 7 (CCS7) [11], [12] protocol supported by ITU.

First of all, our network restoration scheme assumes all network components, branches and subsystems are under constant surveillance, and any developing error or fault will be detected almost instantly. For example, partial failure in the erbium doped fiber amplifier can be detected immediately by embedding in-line fiber Bragg gratings [13]. The fault information will then be sent to hierarchical management nodes via a separate electrical signaling network based on CCS7. To minimize the system downtime, alternate pathways for traffic re-routing away from the point of failure will be computed in advance. If the failure cannot be corrected by backup feature, the network manager will then reset the lightpaths in accordance with the precomputed alternate pathways to restore the network.

Second, for complete network restoration, we present two algorithms generated from integer linear programming but based on different operating principles. The first restoration scheme is link-based restoration $(L B R)$ and is based on identifying all available bypass lightpaths around the failed link. The second scheme is the source-based restoration $(S B R)$ and is generated by considering all possible lightpaths between the source and its destination. While the SBR scheme is more complex than the LBR scheme, it has the advantage of ensuring fairness, which cannot be dealt with by the LBR scheme easily. Unfair situations will arise when lightpath demands is very high for some source- destination node pairs but small for some others. Although we only treat single link failure, the algorithms can easily be extended to cover the case with multiple-link failures. Neither scheme, however, consider wavelength conversion in the network. Note also that the number of WDM channels carried in each fiber is kept constant. For illustrations, our algorithms are applied to the NSFNET backbone network and the European optical network.

This paper is divided as follows. Section II will discuss the supervisory management and protocol design. Section III will present the restoration algorithms, their performances, and case studies. Section IV will discuss the completely restorable network planning. Section V will conclude this work. 


\section{SuPERVISORY MANAGEMENT HiERARChy AND PROTOCOL DESIGN}

\section{A. Supervisory Management Hierarchy}

The supervisory management scheme assumes all fault-identification and recovery functions in the WDM systems are carried out at the component and subsystem levels. The operation status of the components and subsystems will then be pulled or polled periodically by managing nodes arranged in a hierarchical order.

Fig. 1 illustrates the proposed supervisory-management hierarchy for a WDM wavelength routing network. To simplify the structure, each individual link forming the network is considered as lowest layer in the hierarchy, and the switching node at which the link terminates will be assigned as the node manager. The health status of the components and subsystems within that link are reported periodically to the node manager through an independent signaling network. The node managers in turn forward the surveillance information to a dedicated node serving as the network manager, again through the signaling network. A separate node will be assigned as the backup to the network manager. We favor to use automatic correction as extensive as possible at the component and subsystem level without invoking the commands from the managers so as to minimize the system downtime. For any developing fault which cannot be fixed at the component or subsystem level, such as a fiber cut in one of the trunks, the network manager will issue commands to the switching nodes through the signaling network for traffic rerouting based on precomputed lightpaths. Since there is little change in the traffic patterns in the transport network over a short period of time, we can safely assume that precomputed lightpath assignment is adequate to restore the traffic after the link fails.

The transmission of fault information should be via an independently signaling network. This is essential because failure in any transmission link will not affect the transmission of fault information. An extension of the CCS7 is well suited for this purpose. The signaling network consists of signal points (SPs) and signal transfer points (STPs), serving respectively the functions of signaling data sending and receiving, and signaling data routing. To enhance the reliability of the signaling network, each SP is connected to at least two STPs. This built-in redundancy is to avoid traffic congestion and link failure in the signaling network. If the link through STP fails, management messages will be sent via an alternate pathway through the second STP.

The likelihood of partial and complete breakdown of individual system components varies from one type of device to another, and can be caused by failure mechanisms of very different nature. Table I attempts to give a summary of some common optical components with their failure causes, and some of the reported surveillance schemes.

\section{B. Protocol Design}

For detail implementation, the health statuses of the components can be embedded in the signaling information field (SIF) in the message signal units (MSUs) shown in Fig. 2.

The vast capacity in SIF also permits the insertion of network restoration information where lightpaths can be setup

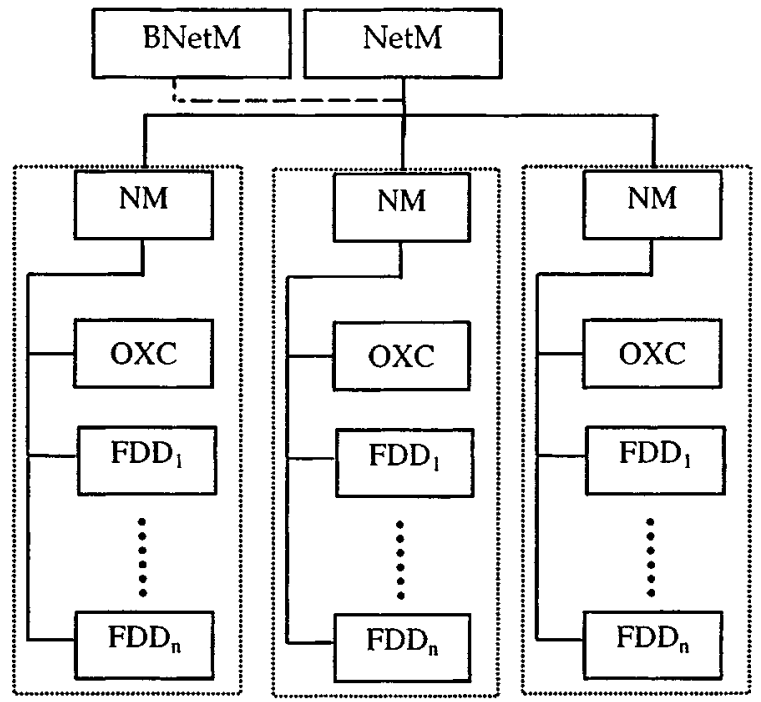

Fig. 1. Network management hierarchy. The WDM wavelength routing network is divided into different branches or links. All link components and subsystem will be monitored, and the switching node at which the fiber terminates will serve as the node manager. The health status will be sent to the node managers and then to the network manager through a separate independent signaling network. NetM-network manager, BnetM-backup network manager, NM-node manager, FDD—fault detection device, and OXC—optical cross-connect.

TABLE I

A Summary of SOME COMMON OPTICAL COMPONENTS WITH THEIR FAILURE CAUSES, AND SOME OF THE REPORTED SuRVEILlanCE SCHEMES. THE QUOTED EXAMPLES ARE BY No MEANS COMPLETE. EDFA-ERBIUM DOPED FIBER AMPLIFIER, OXC-OPTICAL CROSS-CONNECT, ADM-ADD-DROP MULTIPLEXER, WC-WAVELENGTH CONVERTER, AND TX-TRANSMITTER

\begin{tabular}{l|l|l}
\hline $\begin{array}{c}\text { Component } \\
\text { Type }\end{array}$ & \multicolumn{1}{|c|}{ Failure Type } & Ref \\
\hline Fiber & Fiber breaks & {$[14]$} \\
\hline EDFA & Failure in pump diode & {$[13]$} \\
\cline { 2 - 3 } & Power non-equalization & {$[15]$} \\
\hline OXC and & $\begin{array}{l}\text { Errors in wavelength } \\
\text { touting caused by partial } \\
\text { or complete failures in } \\
\text { switches }\end{array}$ & {$[16]$} \\
\cline { 2 - 3 } & $\begin{array}{l}\text { Wavelength mismatch } \\
\text { caused by temperature } \\
\text { change in the } \\
\text { environment }\end{array}$ & {$[17]$} \\
\hline WC & Wavelength mismatch & {$[17]$} \\
\hline Tx & $\begin{array}{l}\text { Wavelength drifting due } \\
\text { either to a breakdown in } \\
\text { thermal control or aging }\end{array}$ & {$[18]$} \\
\hline
\end{tabular}

through wavelength reassignments and through resettings of the switches in the OXC to bypass the failed component or link. The source and destination nodes, and all routing nodes along its pathway have to be informed by the network manager node. These messages can also be embedded in the SIF subfield, as shown in Fig. 3.

We proposed to assign an node identifier (NI) subfield to identify which of the OXC in the network needs to be reconfigured. Using 32 bits to denote NI, the maximum value of NI is 
4 billion. This is large enough to support any backbone network. A 4-byte working lightpath information (WI) subfield is used to describe a lightpath being released from the network. This subfield will contain all necessary information related to the input and output ports of all nodes traversed by the selected lightpath. Likewise, restoration lightpath information (RI) subfield is reserved for the restoration lightpaths. As each node may serve as a source or a destination to multiple lightpaths, the wavelength reassignment information will be embedded in the $M I$ (wavelength reassignment information) subfield.

In the event that all these information exceeds the allowable capacity in the MSU, more than one MSU can be used to carry these messages. We assume that when the working lightpaths are set up, the restoration lightpaths are already computed as well by the restoration algorithm described in Section III. The restoration lightpaths are stored in the NetM and BnetM. When network failures are detected by FDD, NM reports them to the NetM, which in turn retrieves the lightpaths from the restoration lightpath database and sends the restoration message to the corresponding NM.

\section{COMPLETEly RESTORABLE Routing AlgORITHMS}

In this section, we present two algorithms based on integer linear programming. We consider the application of completely restorable routing algorithms for national scale backbone networks. These networks carry traffic from millions of users and so aggregate traffic behavior, like average traffic rate between each node pair, can be derived from past traffic statistics. We assume that the traffic demand matrix denotes the average traffic rate between every node pair.

Reference [9] presents some algorithms which finds lightpaths without the consideration of network failure. Here, we extend their work to find the optimal routing assignment that is completely restorable even if network failure occurs. Two types of lightpaths, namely, working lightpaths and restoration lightpaths, were considered in both algorithms. The working lightpaths are those used under normal operating conditions. Corresponding to each working lightpath, there is a restoration lightpath which will be activated in the event of a failure along the fiber link or segment which cannot be recovered automatically. Thus, restoration of all working lightpaths is guaranteed.

The first restoration scheme is LBR and is based on identifying all available bypass lightpaths around the failed link. The second scheme is the SBR and is generated by considering all possible lightpaths between the source and its destination. The algorithms are modeled by integer linear programming. The parameters and their descriptions are given in Table II.

\section{A. Objective Function and Demand Constraint}

Under the link-based or source-based restoration requirements to be described below, the problem is to find a set of $w_{p, k}$ that minimizes $z$, defined as the maximum insufficient

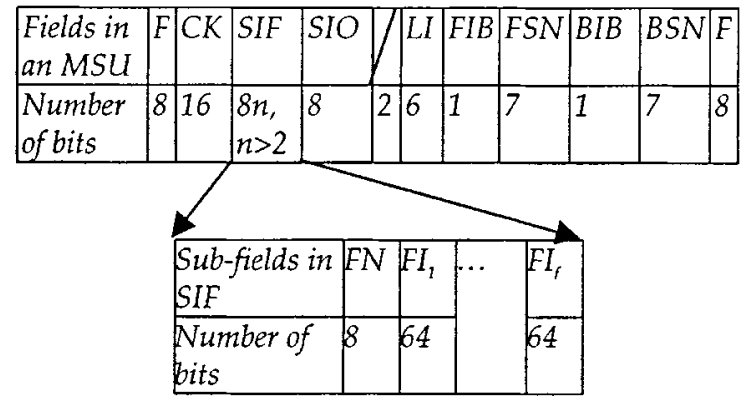

Fig. 2. MSU of CCS7. The SIF subfield embeds the surveillance information of all network components and branches. $F$ : a marker with sequence "01 111110 " to signify the beginning and ending of an MSU; BSN: backward sequence number; $B I B$ : backward indicator bit; $F S N$ : forward sequence number; $F I B$ : forward indicator bit. The BSN, BIB, FSN and FIB are designed for error correction, message sequence control, confirmation and re-transmission. $L I$ : length indicator specifying the length of SIF. $C K$ : error checking code; SIO: service indicator octet; FN: number of failed elements; $F I_{j}$ : information of the $j$ th failed component.

\begin{tabular}{|l|l|}
\hline Sub-field in SIF & Number of bits \\
\hline$N I$ & 32 \\
\hline$W N$ & 8 \\
\hline$W I_{l}-W I_{w}$ & 32 \\
\hline$R N$ & 8 \\
\hline$R I_{I}-R I_{r}$ & 32 \\
\hline$M N$ & 8 \\
\hline$M I_{l}-M I_{m}$ & 32 \\
\hline
\end{tabular}

Fig. 3. Lightpath restoration embedded in the SIF subfields. NI: node identifier specifying which OXC needs to be reconfigured; $W N$ : the number of working lightpaths released due to component or link failure; WI: total of $w$ 32-bit subfields containing information on each released lightpath; $R N$ : number of restoration lightpaths; RI: total of $r$ 32-bit subfields describing the restoration lightpaths; $M N$ : number of wavelength needs to be reassigned; $M I$ : total of $m$ 32-bit subfields describing the wavelength reassignments.

TABLE II

SYMBOLS AND THEIR CORRESPONDING DESCRIPTIONS USED IN THE ALGORITHMS

\begin{tabular}{l|l}
\hline \multicolumn{1}{|c|}{ Symbol } & \multicolumn{1}{|c}{ Description } \\
\hline$K$ & $\begin{array}{l}\text { Number of wavelength channels on each } \\
\text { fiber }\end{array}$ \\
\hline$N$ & Number of network nodes \\
\hline$E$ & $\begin{array}{l}\text { Number of source-destination pairs with } \\
\text { traffic demands }\end{array}$ \\
\hline$d_{s, t}$ & Number of edges in the network; \\
\hline$f_{e}$ & $\begin{array}{l}\text { Number of lightpaths required between the } \\
\text { node pair }(s, t), \text { where } s \text { and } t=1,2 \ldots N ;\end{array}$ \\
\hline$w_{p, k}$ & $\begin{array}{l}\text { Number of fibers on edge } e, \text { where } e=1,2, \\
\ldots E\end{array}$ \\
\hline
\end{tabular}

allocation of lightpaths among all node pairs. Mathematically, $z$ is expressed as

$$
z=\max _{(s, t)}\left[d_{s, t}-\sum_{\substack{\text { all k. } \\ p \text { incident } \\ \text { on } s, t}} w_{p, k}\right] .
$$


This objective function is preferred to that by simply maximizing the total number of lightpaths as the latter could lead to unfair allocation of lightpaths to node pairs with different demands. As there is no need to assign more lightpaths than what is demanded, and as $z$ is the maximum unsatisfied demand amount, the demand constraint for both LBR and SBR is as follows.

C1. Demand Constraint: The total number of working lightpaths originating from node $s$ to node $t$ must be between $d_{s, t}-z$ and $d_{s, t}$, or

$$
d_{s, t}-z \leq \sum_{\substack{\text { all } k, p \text { incident } \\ \text { on } s, t}} w_{p, k} \leq d_{s, t} .
$$

\section{B. Link-Based Restoration Algorithm}

For LBR, we introduce variable $b_{e, p, k}$ which denotes the number of the bypass lightpaths set up on route $p$ using wavelength channel $\lambda_{k}$ when link $e$ fails. Let a working lightpath be denoted by $\left(w_{1}, w_{2}, \ldots w_{n}\right)$, where $w_{i}$ is the $i$ th link of the lightpath. Suppose a failure occurs on link $e_{f}$ and the bypass lightpath for this link is $\left\{b_{1}, b_{2}, \ldots b_{m}\right\}$, then the restored working lightpath can be described as $\left\{w_{1}, w_{2}, \ldots w_{f-1}, b_{1}, b_{2}, \ldots b_{m}, w_{f+1}, w_{n}\right\}$. Loops, if exist in the restored lightpath, have to be removed. For the LBR algorithm, the following two additional constraints have to be satisfied.

C2. LBR Lightpath Bypass Constraint: For each $\lambda_{k}$ in a failed link $e^{*}$, the number of lightpaths that bypass $e^{*}$ and are on path $p^{*}$ must be no smaller than the number of working lightpaths that pass through $e^{*}$ and are on the path $p$, i.e.

$$
\sum_{p^{*} \text { bypasses } e^{*}} b_{e^{*}, p^{*}, k} \geq \sum_{\substack{p \text { passes } \\ \text { through } e^{*}}} w_{p, k} .
$$

C3. Capacity Constraint: Under normal situation, the total number of working lightpaths passing through link $e$ on wavelength channel $\lambda_{k}$ must be no larger than $f_{e}$. Therefore, we have

$$
\sum_{\substack{p \text { passes } \\ \text { through } e}} w_{p, k} \leq f_{e} .
$$

Under a link failure situation, for each link $e$ on the bypass lightpath of failed link $e^{*}$, the total number of working lightpaths plus the number of bypass lightpaths on $e$ must be no larger than $f_{e}$.

$$
\sum_{\substack{p^{*} \text { bypasses } \\ e^{*} \text { and passes } \\ \text { through } e}} b_{e^{*}, p^{*}, k}+\sum_{\substack{p \text { passes } \\ \text { through } e}} w_{p, k} \leq f_{e} .
$$

\section{Source-Based Restoration Algorithm}

For SBR, we introduce variable $r_{e^{*}, p^{*}, k}$ denoting the number of the restoration lightpaths established on route $p^{*}$ for wavelength channel $\lambda_{k}$ when link $e^{*}$ fails. The constraints for SBR are the following.
C4. SBR Restoration Lightpath Constraint: For each wavelength channel, $\lambda_{k}$ in a failed link $e^{*}$, the number of restored lightpaths must be no smaller than the number of working lightpaths originally passing through $e^{*}$.

$$
\sum_{\substack{p^{*} \text { does not } \\
\text { pass through } \\
\text { and incident on } \\
(s, t)}} r_{e^{*}, p^{*}, k} \geq \sum_{\begin{array}{c}
p \text { pass } \\
\text { through } e^{*} \\
\text { and incident on } \\
(s, t)
\end{array}} w_{e^{*}, p, k} .
$$

C5. Capacity Constraint: Under normal situation, the capacity constraint is the same as that of LBR, i.e.

$$
\sum_{\substack{p \text { passes } \\ \text { through } e}} w_{p, k} \leq f_{e} .
$$

Under a link failure situation, for each failed link $e^{*}$ and for each $\lambda_{k}$, the total number of restoration and working lightpaths on any other healthy link $e$ should not exceed $f_{e}$.

$$
\sum_{\substack{p^{*} \text { does not } \\
\text { pass through } \\
e^{*} \text { but } e}} r_{e, p^{*}, k}+\sum_{\begin{array}{c}
p \text { pass } \\
\text { through } e \\
\text { but not } e^{*}
\end{array}} w_{p, k} \leq f_{e}
$$

\section{Case Studies}

Computer programs were developed for the two restoration algorithms using the IBM OSL linear integer programming software. Our algorithms were tested for the three existing networks and their performances were compared using a Pentium PC.

In case I, we use the 11-node network well cited in restoration studies [5]-[7] and assume a randomly generated traffic demand [20]. The total demanded lightpaths is 38 . The two algorithms are compared for $K=2$ and $K=4$. When $K=2$, the numbers of lightpaths set up by the two algorithms are 16 and 18 . When $K=4$, the numbers of lightpaths found by both algorithms are 32. Fig. 4 shows an example of restoration lightpaths set up by the LBR and SBR algorithms respectively. We shall discuss on case I after presenting case II as the conclusions are similar. The computation times for LBR and SBR are 30 and $45 \mathrm{~min}$, respectively.

Case II is the study of the restoration on the 16-node NSFNET T1 backbone network [21]. The lightpath demand shown in Table III is derived from Internet traffic statistics. We assume 10 fibers are installed on each edge. The total number of lightpaths demand is 102. The two algorithms are compared for $K=2$ and $K=4$. When $K=2$, the numbers of lightpaths set up by the LBR and SBR are 42 and 48, respectively. When $K=4$, the numbers are 82 and 83 . The assignment of lightpaths for SBR is shown in the last column of Table III. It is seen that the maximum number of unsatisfied demand is three in this case.

The running time of SBR algorithm is much longer than that of LBR in both cases. For $K=2$, the computation time for LBR and SBR are 120 and $200 \mathrm{~min}$, respectively. For $K=$ 4, the times are 180 and $900 \mathrm{~min}$. As shown by the figures, a small increase in the number of variables and constraints in SBR leads to a substantial increase in computation time. As expected, the performance of SBR is better than that of LBR in general. However, as $K$ increases from 2 to 4 , the percentage increase in total restorable lightpath diminishes (42 increases to 82 for 


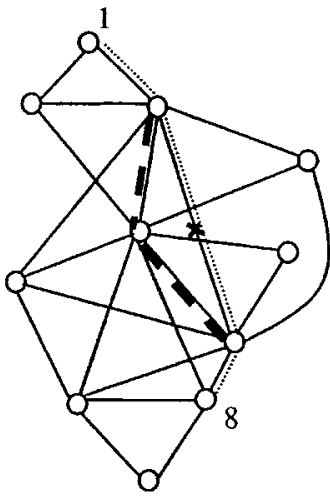

(a) Link-based

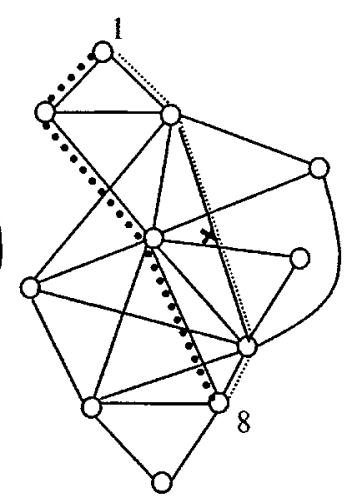

(b) Source-based

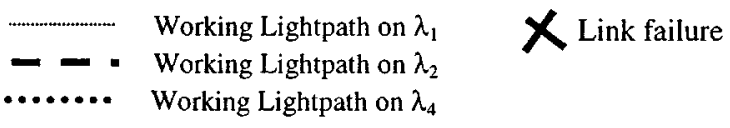

Fig. 4. Restoration for the working lightpath between node 1 and node 8 .

LBR and 48 increases to 83 for SBR). This shows that in order to reduce wavelength conflicts, for setting up restorable lightpaths, we can either increase the number of topological paths as in SBR or simply increase $K$, the wavelength channels in a fiber.

As a general guideline, when $K$ is small, SBR can give better performance in acceptable run time. On the other hand, when $K$ is large, LBR is preferred as it can give virtually the same performance with significantly shorter computation time. Moreover, for a large network, LBR may be the only feasible algorithm to use.

In case III, this is the study of LBR on the European Optical Network [11]. The randomly generated demand matrix contains 70 node pairs with traffic demands, and the total number of lightpaths demanded is 283. Computer simulation is performed for $K=4,8$ and 16. The results are shown in Table IV.

This table shows that $K=16$ can barely satisfy all the traffic demand in this case. As the algorithm will not assign more lightpath than what is needed, this is the optimal solution. Notice that as $K$ increases from 4 to 8 the total lightpath is more than doubled showing the effect of reduced wavelength conflict as $K$ increases.

As for dependency of computational time complexity on $K$, we found that when $K=4$ and 8 , the solution can be found in 180 and $500 \mathrm{~min}$, respectively. However, $30 \mathrm{~h}$ are required when $K=16$. Since the integer program is solved by the Branch and Bound method, a number of integer solutions are generated during the process. It is observed that there are significant improvements of the integer solutions in the first 4 hours, very little improvement is achieved in the next 21 hours and no improvement is obtained in the last five hours of computation. What is comforting is that there is very little difference between the final integer solution and the upper bound of the integer solution obtained by removing the integer constraints.

\section{Optimal Completely Restorable Network Planning}

There are different types of backbone network planning. Type 1 assumes that the network is to be built from scratch. This type
TABLE III

TRAFFic DEMAND AND AssignMENT RESUlts FOR SBR with $K=4$

\begin{tabular}{l|l|l|l}
\hline Source & $\begin{array}{l}\text { Destin } \\
\text { ation }\end{array}$ & $\begin{array}{l}\text { Traffic } \\
\text { Demand }\end{array}$ & $\begin{array}{l}\text { Assigned } \\
\text { Lightpath }\end{array}$ \\
\hline WA & NE & 3 & 3 \\
\hline CA1 & WA & 2 & 2 \\
\hline CA1 & GA & 4 & 2 \\
\hline CA2 & IL & 21 & 20 \\
\hline UT & CA1 & 3 & 1 \\
\hline UT & PA & 1 & 1 \\
\hline TX & NY & 7 & 5 \\
\hline NE & CO & 1 & 1 \\
\hline I & MD & 9 & 9 \\
\hline PA & WA & 3 & 3 \\
\hline MI & CO & 7 & 4 \\
\hline GA & CA1 & 2 & 2 \\
\hline NJ & NE & 5 & 2 \\
\hline MD & WA & 3 & 2 \\
\hline MD & NJ & 7 & 7 \\
\hline GA & NE & 6 & 3 \\
\hline PA & CA1 & 14 & 12 \\
\hline CO & NY & 4 & 4 \\
\hline & \multicolumn{2}{|l}{}
\end{tabular}

TABLE IV

COMPUTER SiMULATION FOR EUROPEAN OPTICAL NETWORK USING $K=4,8$, AND 16

\begin{tabular}{l|l}
\hline $\begin{array}{l}\text { Wavelength } \\
\text { channels in } \\
\text { each fiber }(K)\end{array}$ & $\begin{array}{l}\text { Total number of } \\
\text { restorable lightpaths }\end{array}$ \\
\hline 4 & 65 \\
\hline 8 & 142 \\
\hline 16 & 283 \\
\hline
\end{tabular}

of network can be planned by using the minimum variance algorithm [22]. Type 2 assumes a network with traffic demand exceeding its capacity. The planning is to decide where to add capacity to satisfy this growing demand. Type 3 assumes that the working capacity is given and only the spare capacity has to be planned [5]-[7]. The optimal completely restorable network planning algorithms presented in this section are the combination of type 2 and 3 . In many developed countries, a large quantity of dark fibers has already been laid by telecommunication companies. Adding capacity simply means the adding of transmitters and receivers of appropriate wavelengths at both ends of the fiber links. In this section, we focus on the network-planning problem for networks that can restore all working lightpaths upon any single link failure. The solution of the completely restorable planning problem consists of the number of fibers needed on each edge and the working and restoration lightpath assignments.

Due to their similarities, the SBR and LBR algorithms can be easily transformed to the source-based restoration planning and link-based restoration planning algorithms. Let the lightpath demand between every node pair be given, say, by a certain forecasting method. Let $f_{e}^{\prime}$ be the number of fibers desired on edge $e, f_{e}$ be the number of existing fibers on edge $e$ and $c_{e}\left(f_{e}^{\prime}-f_{e}\right)$ 
be the upgrading cost on edge $e$. The LBR network-planning problem is to minimize the total upgrading cost $C$ defined as

$$
C=\sum_{e} c_{e}\left(f_{e}^{\prime}-f_{e}\right)
$$

with respect to $f_{e}^{\prime}$ subject to constraints 2, 3 (or 4, 5 for SBR network planning) and,

C6. Network Planning Demand Constraint: For any node pair $(s, t)$, the sum of all working lightpaths from $s$ to $t$ should not be smaller than $d_{s, t}$, i.e.

$$
\sum_{\substack{\text { all } k, p \text { incident } \\ \text { on } s, t}} w_{p, k} \geq d_{s, t} .
$$

\section{CONCLUSION}

In conclusion, we have presented here a supervisory management and two restoration algorithms tailored for all-optical wavelength routing networks. The supervisory management scheme uses a hierarchical approach with a network manager supervising all node managers, each of which monitors the health statuses of all network components and subsystems of that link. We suggest using an independent signaling network to transmit the surveillance and management information to guarantee the reliability of fault detection and reporting. We also suggest an extension to the existing CCS7 protocol to include the surveillance information.

Two lightpath restoration algorithms were developed. The first approach uses LBR and assumes at finding bypass lightpaths around the failed link, while the second approach uses SBR and focuses on finding bypass lightpaths between the source and destination nodes. The performances of both algorithms were compared in three networks commonly cited for testing purposes. In general, SBR performs better than LBR, but requires much longer computation time. But as $K$, the number of wavelengths on each fiber, increases, their performance difference is significant. Also, for larger networks, LBR is the only feasible algorithm due to computational complexity. The two algorithms can also be easily adapted for planning completely restorable networks as shown in Section IV.

\section{REFERENCES}

[1] J. Lightwave Technol., vol. 11, May/June 1993.

[2] Y. Dubinsky and A. Segall, "A flexible rerouting protocol in ATM networks," Proc. INFOCOM Conf., vol. 3, pp. 1488-1496, 1999.

[3] H. Hwang, K. Kim, Y. Choi, C. Kim, and S. Ahn, "Virtual backup network for broadband network restoration," Proc. IEEE ICC Conf., vol. 2, pp. 1129-1133, 1998.

[4] E. Chow, C, Bicknell, J. McCaughey, and S. Syed, "A fast distributed network restoration algorithm," in Twelfth Annu. Int. Phoenix Conf. Comput. Commun., 1993, pp. 261-267.
[5] H. Sakauchi, Y. Nishmura, and S. Hasegawa, "A self-healing network with an economical spare-channel assignment," Proc. IEEE GLOBECOM Conf., vol. 1, pp. 438-443, 1990.

[6] M. Herzberg, "A decomposition approach to assign spare channels in self-healing networks," in Proc. IEEE GLOBECOM Conf., 1993, pp. $1601-1605$

[7] M. Herzberg and S. J. Bye, "An optimal spare-capacity assignment model for survivable networks with hop limit," IEEE Commun. Mag., pp. 1601-1606, 1994.

[8] R. Ramaswami and K. N. Sivarajan, "Optimal routing and wavelength assignment," in Proc. IEEE INFOCOM Conf., 1994, pp. 78-88.

[9] N. Wauters and P. Demesster, "Design of the optical path layer in multiwavelength cross connected networks," IEEE J. Select. Areas Commun., vol. 14, pp. 881-892, 1996.

[10] B. Van Caenegem, W. Van Parys, F. De Truch, and P. M. Demeester, "Dimensioning of survivable WDM networks," IEEE J. Select. Areas Commun., vol. 16, pp. 1146-1157, 1998.

[11] C. Bush and A. Smith, "Software planning tool for CCS7 network with an emphasis on routing management," Proc. IEEE ICC Conf., vol. 2, pp. 626-630, 1997.

[12] T.-H. Wu, J. C. McDonald, T. P. Flanagan, and K. Sato, "Integrity of public telecommunication networks," IEEE J. Select. Areas Commun., vol. 12, pp. 1-4, 1994.

[13] C. K. Chan, F. Tong, L. K. Chen, and D. Lam, "An in-service passive surveillance system for optically amplified branched optical networks," Electron. Lett., vol. 33, no. 9, pp. 795-797, Apr. 1997.

[14] C. K. Chan, F. Tong, L. Chen, J. Song, and D. Lam, "A practical passive surveillance scheme for optically amplified passive branched optical networks," IEEE Photon. Technol. Lett., vol. 9, pp. 526-528, Apr. 1997.

[15] A. Chraplyvy, R. Tkach, K. C. Reichmann, P. D. Magill, and J. A. Nagel, "End-to-end equalization experiments in amplified WDM lightwave systems," IEEE Photon. Technol. Lett., vol. 5, pp. 428-429, Apr. 1994.

[16] C. K. Chan, E. Kong, F. Tong, and L. K. Chen, "A novel path supervisory scheme for optical cross-connects in all-optical transport networks," IEEE Photon. Technol. Lett., vol. 10, pp. 899-901, June 1998.

[17] — "A novel wavelength matching scheme for wavelength grating routers in all-optical transport networks," Electron. Lett., vol. 34, no. 5, pp. 490-491, 1998.

[18] H. Okamoto, H. Yasaka, Y. Yoshikuni, and L. Oe, "Suppression of wavelength drift and thermal crosstalk in a tunable duplex integrated light source," Proc. IEEE LEOS Conf., vol. 2, pp. 297-298, 1995.

[19] R. Travis, Signaling System \#7. New York: McGraw-Hill, 1995.

[20] C. C. Hui, "Design, protocol and routing algorithms for survivable alloptical networks," M.Phil. thesis, Dept. of Information Engineering, The Chinese University of Hong Kong, Hong Kong, 1998.

[21] B. Mukherjee, Optical Communication Networks. New York: McGraw-Hill, 1997.

[22] C. M. Lee, C. C. Hui, F. Tong, and T. S. Yum, "Network dimensioning in WDM based all-optical network," Proc. IEEE GLOBECOM Conf., vol. 1, pp. 328-333, 1999.

Frank Tong (M'90-SM'95), photograph and biography not available at the time of publication.

Tak-Shing Yum (S'76-A'78-SM'86), photograph and biography not available at the time of publication.

Chi-Chun, photograph and biography not available at the time of publication. 